\title{
Ommaya Reservoir Tap
}

National Cancer Institute

\section{Source}

National Cancer Institute. Ommaya Reservoir Tap. NCI Thesaurus. Code C38077.

A procedure to remove cerebrospinal fluid from the reservoir to detect any abnormal cells. 